\title{
Forensic use of Y-chromosome DNA: a general overview
}

\author{
Manfred Kayser ${ }^{1}$
}

Received: 5 February 2017 / Accepted: 8 March 2017 / Published online: 17 March 2017

(c) The Author(s) 2017. This article is an open access publication

\begin{abstract}
The male-specific part of the human Y chromosome is widely used in forensic DNA analysis, particularly in cases where standard autosomal DNA profiling is not informative. A Y-chromosomal gene fragment is applied for inferring the biological sex of a crime scene trace donor. Haplotypes composed of Y-chromosomal short tandem repeat polymorphisms (Y-STRs) are used to characterise paternal lineages of unknown male trace donors, especially suitable when males and females have contributed to the same trace, such as in sexual assault cases. Y-STR haplotyping applied in crime scene investigation can (i) exclude male suspects from involvement in crime, (ii) identify the paternal lineage of male perpetrators, (iii) highlight multiple male contributors to a trace, and (iv) provide investigative leads for finding unknown male perpetrators. Y-STR haplotype analysis is employed in paternity disputes of male offspring and other types of paternal kinship testing, including historical cases, as well as in special cases of missing person and disaster victim identification involving men. Y-chromosome polymorphisms are applied for inferring the paternal bio-geographic ancestry of unknown trace donors or missing persons, in cases where autosomal DNA profiling is uninformative. In this overview, all
\end{abstract}

This article is dedicated to the 25 years of forensic

Y-chromosome research and applications, since the description of the first Y-chromosomal STR marker published in this journal (Roewer et al. Hum Genet 1992; 89:389-94), and its immediate use in forensic casework (Roewer and Epplen Forensic Sci Int 1992; 53:163-171).

Manfred Kayser

m.kayser@erasmusmc.nl

1 Department of Genetic Identification, Erasmus MC University Medical Center Rotterdam, PO Box 2040, 3000

CA Rotterdam, The Netherlands different forensic applications of Y-chromosome DNA are described. To illustrate the necessity of forensic Y-chromosome analysis, the investigation of a prominent murder case is described, which initiated two changes in national forensic DNA legislation both covering Y-chromosome use, and was finally solved via an innovative Y-STR dragnet involving thousands of volunteers after 14 years. Finally, expectations for the future of forensic Y-chromosome DNA analysis are discussed.

\section{Making the case with a case}

May 30, 1999, Queensday in The Netherlands: As every year, the Dutch were celebrating their Queen's birthday with concerts, flea markets, and public and private parties all over the country. Marianne Vaatstra, a 16-year-old girl from the small village Zwaagwesteinde in the province Friesland, went for partying to the nearby village Kollum, from where she never returned home alive. On her bicycle ride back home at night, she was raped and murdered nearby the village Veenklooster, with her throat being slit, and traces of semen found in and on her body. No human eyewitness was available. No hit of the standard autosomal DNA profile obtained from the semen stains was found in the national criminal offender DNA database, which started in 1997 and, therefore, only included a few hundred persons by mid-1999.

A suspect from Zwaagwesteinde was arrested weeks later, but got released soon after, because his standard autosomal DNA profile did not match the one from the semen trace. Due to the murder scene's location in close proximity to a political asylum seeker centER, the investigation also focused on the asylum seekers from this center. A man from Iraq, who left the center in the 
night of the murder and, therefore, raised suspicion, was tracked down by INTERPOL in Istanbul, but found innocent because of his non-matching standard autosomal DNA profile; as was a man from Afghanistan. By December 1999, approximately 150 persons, whom the investigators somehow linked with the case (but without enough evidence to make them case suspects), were voluntarily asked for a DNA sample; none of their standard autosomal DNA profiles matched the one obtained from the semen trace.

Soon after the crime happened, the local population strongly expressed its belief that the perpetrator must be one of the asylum seekers from the center, if only because of the assumed to be non-European manner of slit-throat murder. This led to serious conflicts between the local population and asylum seekers in the center as well as between the local population and police authorities. In the difficult situation of increasing social unrest, the public prosecutor in charge of the case turned to Peter de Knijff from the Forensic Laboratory for DNA Research (FLDO), Department of Human Genetics, Leiden University Medical Center. Ordered by the public prosecutor, FLDO obtained a Y-chromosome STR profile from the semen trace to infer the trace donor's paternal bio-geographic ancestry. By comparing it with those stored in the Y-chromosome Haplotype Reference Database (YHRD) (www.yhrd.org), as well as others (published and unpublished) available to him, de Knijff concluded that the semen donor's paternal ancestors are likely to originate from North-western Europe. With this result, it became clear to the investigators that they should rather widen their search for the unknown perpetrator among the Dutch European population. Although many local people were still reluctant to believe that the murderer was one of them, the results of this Y-chromosome bio-geographic ancestry test calmed down some of the social unrest in the region. However, the perpetrator was not found, and the Vaatstra case became a cold case for many years, until a different forensic use human Y-chromosome DNA eventually allowed for solving this murder case, albeit not before 14 years after it occurred.

This forensic use of $\mathrm{Y}$ chromosome DNA was remarkable in two ways. First, although, at that time, Y-STR profiling for paternal lineage identification was already introduced to forensics, for which Peter de Knijff together with Lutz Roewer from the Institute of Legal Medicine, Charité University Medicine Berlin were the leading scientists (de Knijff et al. 1997; Kayser et al. 1997, Roewer et al. 1992, 1996; Roewer and Epplen 1992), its forensic use for biogeographic ancestry inference was not. Second, at that time in The Netherlands, forensic DNA analysis was regulated by a law from 1994, under which only autosomal STR profiling was legally allowed, but DNA inference of bio-geographic ancestry was not.
The investigation of the Vaatstra case is unique in the way that it stimulated two national law adaptations, both covering the forensic use of Y-chromosome DNA, albeit for different purposes. In 2003, likely stimulated by the Vaatstra case and the previous attempts to solve it including the illegally applied Y-chromosome ancestry testing, the Dutch parliament approved the first adaptation of the forensic DNA legislation. This law allows and regulates the forensic use of DNA information regarding bio-geographic ancestry and externally visible characteristics for investigative intelligence purposes to find unknown perpetrators of crime that cannot be identified by any other means. Moreover, in April 2012, the Dutch DNA legislation was adapted for a second time, allowing the forensic use of DNA for familial searching. Familial searching typically refers to the use of DNA evidence to find in criminal offender or suspect databases relatives of unknown perpetrators, whose standard autosomal DNA profiles are not yet included in such database and who, therefore, cannot be identified with DNA directly.

Two active ways of DNA-based familial searching regulated by this law were generally suitable to the Vaatstra case, and were thus applied to the case soon after the law adaptation was put in place. The first is searching the standard autosomal STR profiles of known offenders stored in the national DNA database for those that show strong similarity to the one from the crime scene trace. This approach can highlight close relatives of an unknown perpetrator being already included in the DNA database, which provides that investigative leads to eventually find the unknown perpetrator not yet included in the DNA database. Because of the use of autosomal STRs in standard DNA profiling, this approach is most suitable to trace close relatives (parents, children, and siblings). Distant relatives are difficult, if not impossible, to be traced with autosomal STRs due to occurring DNA recombination events that produce dissimilarities with every subsequent generation. Applying this approach in the Vaatstra case in 2012, when 142,120 persons were included in the national offender DNA database, revealed 121 men for whom an increased probability to be related with the unknown perpetrator was estimated.

Next, the special force team from police and prosecution, including forensic coordinator Ron Rintjema and tactical coordinator Jelle Tjalsma, in collaboration with Charissa van Kooten and Arnoud Kal from the Netherlands Forensic Institute (NFI) performed Y-STR profiling in selected men included in the national DNA database. In The Netherlands, DNA samples of persons from the national DNA database are kept, instead of being destroyed after standard DNA profiling as in some other countries. This is because the investigative use of the DNA samples from persons whose STR profile is stored in the national DNA database, involving additional DNA 
testing, is legally allowed. For the purpose of familial searching in the Vaatstra case, DNA samples from males included in the DNA database were selected for Y-STR analysis based on the following criteria: (i) they were previously identified as potential relatives via familial searching with autosomal STR profiling (121 males), (ii) they were born in or resident of the area in which the crime was committed (421 males), (iii) they carry regionspecific surnames-present in the area where the crime was committed, but rare in The Netherlands as a whole (260 males). The latter criterion was applied because in patrilineal societies, as all European populations are, surnames and Y-chromosomes follow the same paternal mode of inheritance (see below). However, Y-STR profiling of these 802 criminal offenders did not reveal any complete or close match with the semen trace. This finding led to the conclusion that no close or distant paternal male relative of the unknown murderer of Marianne Vaatstra was included within that group of selected persons from the DNA database.

The second way of legally allowed familial searching is large-scale, voluntary DNA mass screenings (also called DNA dragnets) in the restricted geographic region where the crime occurred, assuming that the perpetrator does not participate. This is only allowed under certain circumstances such as serious crime leading to many years of imprisonment, and is particularly meant as last resort to solve cold cases where all other attempts have already failed (including the first described approach of familial search). From the tactical police investigation in the Vaatstra case, it was concluded that the perpetrator likely comes from the region. In September 2012, a large-scale DNA dragnet was decided as last resort to solve the Vaatstra case. More than 7600 men who lived in the region $5 \mathrm{~km}$ around the murderer site were invited to voluntarily provide a cheek swab sample for DNA analysis, and more than 6600 local men (87\%) participated. Importantly, instead of using standard autosomal STR profiling, the special force team together with the NFI decided to apply Y-STR profiling in this DNA dragnet. Under the assumption that the unknown male perpetrator himself will not participate, it makes perfect sense from a scientific and policing perspective to carry out Y-STR profiling to find a male relative of the perpetrator, who, in turn, can guide the investigation to find the non-participating perpetrator. This is because Y-STR profiling in principle allows for highlighting all participating paternal male relatives of an unknown male perpetrator, close and distant ones, who typically share the same Y-STR profile, whereas autosomal STR profiling can only trace close relatives. The regional population and, thus, all volunteers participating in the dragnet were well informed by the authorities on the content and consequences of such
Y-STR-based kinship approach via distributed brochures, leaflets, and a dedicated Website.

Y-STR profiling at 17 Y-STR markers using the commercial AmpFlSTR ${ }^{\circledR}$ Yfiler $^{\circledR}$ kit (Thermo Fisher Scientific) (Table 1) was applied. Remarkably, however, instead of performing Yfiler analysis in all 6600 samples, which is time, labour, and resource intensive, the special force team in collaboration with the NFI applied a more effective approach. After the NFI had done Y-STR analysis in samples from the first set of 81 volunteers, allocated in the first collection box, already two Y-STR haplotype matches with the semen trace were obtained. Although, subsequent autosomal STR profiling excluded both men as likely suspects, this was a breakthrough finding. The Y-STR profile from the semen trace was so rare that it had not been ever recorded in any reference databases worldwide (including YHRD and a large unpublished Dutch Y-STR reference database); however, it showed up twice among the first 81 regional men analysed. By luck and thanks to the use of Y-STR profiling, the team had traced the paternal family of the unknown perpetrator after having analysed the first 81 regional men only. This result confirmed the previous assumption that led to the regional DNA dragnet, that the unknown perpetrator likely was a local man; at least his close and/or distant paternal relatives were, indeed, living in this area.

Moreover, instead of continuing with Y-STR profiling systematically in a box-by-box manner until all 6600 volunteers were analysed, the special force team then performed genealogy research in public registry archives on the two Y-STR matching volunteers. What they found was that these two men, who had different surnames, shared the same paternal ancestor at a time before the Dutch were forced to have their surnames registered during the Napoleon occupation. This explains why they share the same Y-STR haplotype but carry different surnames. The team then used this knowledge for effectively prioritizing the subsequent Y-STR analysis. They selected samples from volunteers with these two surnames, which could indicate that they belong to the perpetrator's extended paternal family. By applying this approach, Y-STR profiles were never generated on thousands of collected samples, which saved time, money, and resources. Moreover, this intelligencedriven approach secured the privacy of thousands of volunteers, whose collected DNA samples were never analysed.

As may be expected for a rural area such as Friesland where typically male relatives stay in the region, the team identified several volunteers who matched the Y-STR haplotype from the semen trace. Aiming to further guide the investigation to the perpetrator's close (instead of distant) relatives, it was decided that additional Y-STR markers need to be analysed in the DNA samples from the semen trace as well as from all volunteers with matching 
Table 1 Y-STR markers widely used in forensic DNA analysis

\begin{tabular}{|c|c|c|c|c|c|c|}
\hline \multirow[t]{2}{*}{ Y-STR marker } & \multicolumn{6}{|c|}{ Commercial Y-STR kits/Non-commercial Y-STR sets } \\
\hline & $\begin{array}{l}\text { Minimal } \\
\text { haplotype }^{c}\end{array}$ & $\begin{array}{l}\text { PowerPlex }^{\circledR} \\
\text { Ya }^{\mathrm{a}}\end{array}$ & $\begin{array}{l}\text { AmpFlSTR } \\
\text { Yfiler }^{\circledR b}\end{array}$ & $\begin{array}{l}\text { PowerPlex }^{\circledR} \\
\text { Y23 }^{\text {a }}\end{array}$ & $\begin{array}{l}\text { Yfiler }^{\circledR} \\
\text { Plus }^{\mathrm{b}}\end{array}$ & $\begin{array}{l}\text { RM Y-STR } \\
\text { set }^{c}\end{array}$ \\
\hline DYS19 & $\checkmark$ & $\checkmark$ & $\checkmark$ & $\checkmark$ & $\checkmark$ & \\
\hline DYS385a/b & $\checkmark$ & $\checkmark$ & $\checkmark$ & $\checkmark$ & $\checkmark$ & \\
\hline DYS389I & $\checkmark$ & $\checkmark$ & $\checkmark$ & $\checkmark$ & $\checkmark$ & \\
\hline DYS389II & $\checkmark$ & $\checkmark$ & $\checkmark$ & $\checkmark$ & $\checkmark$ & \\
\hline DYS390 & $\checkmark$ & $\checkmark$ & $\checkmark$ & $\checkmark$ & $\checkmark$ & \\
\hline DYS391 & $\checkmark$ & $\sqrt{ }$ & $\checkmark$ & $\checkmark$ & $\checkmark$ & \\
\hline DYS392 & $\checkmark$ & $\checkmark$ & $\checkmark$ & $\checkmark$ & $\checkmark$ & \\
\hline DYS393 & $\checkmark$ & $\checkmark$ & $\checkmark$ & $\checkmark$ & $\checkmark$ & \\
\hline DYS437 & & $\checkmark$ & $\checkmark$ & $\checkmark$ & $\checkmark$ & \\
\hline DYS438 & & $\checkmark$ & $\checkmark$ & $\checkmark$ & $\checkmark$ & \\
\hline DYS439 & & $\checkmark$ & $\checkmark$ & $\checkmark$ & $\checkmark$ & \\
\hline DYS448 & & & $\checkmark$ & $\checkmark$ & $\checkmark$ & \\
\hline DYS456 & & & $\checkmark$ & $\checkmark$ & $\checkmark$ & \\
\hline DYS458 & & & $\checkmark$ & $\checkmark$ & $\checkmark$ & \\
\hline DYS635 & & & $\checkmark$ & $\checkmark$ & $\checkmark$ & \\
\hline Y-GATA-H4 & & & $\checkmark$ & $\checkmark$ & $\checkmark$ & \\
\hline DYS481 & & & & $\checkmark$ & $\checkmark$ & \\
\hline DYS533 & & & & $\checkmark$ & $\checkmark$ & \\
\hline DYS549 & & & & $\checkmark$ & & \\
\hline DYS570 & & & & $\checkmark$ & $\checkmark$ & $\checkmark$ \\
\hline DYS576 & & & & $\checkmark$ & $\checkmark$ & $\checkmark$ \\
\hline DYS643 & & & & $\checkmark$ & & \\
\hline DYS449 & & & & & $\checkmark$ & $\checkmark$ \\
\hline DYS460 & & & & & $\checkmark$ & \\
\hline DYS518 & & & & & $\checkmark$ & $\checkmark$ \\
\hline DYS627 & & & & & $\checkmark$ & $\checkmark$ \\
\hline DYF387S1a/b & & & & & $\checkmark$ & $\checkmark$ \\
\hline DYS526a/b & & & & & & $\checkmark$ \\
\hline DYS547 & & & & & & $\checkmark$ \\
\hline DYS612 & & & & & & $\checkmark$ \\
\hline DYS626 & & & & & & $\checkmark$ \\
\hline DYF399S1 & & & & & & $\checkmark$ \\
\hline DYF403S1a/b & & & & & & $\checkmark$ \\
\hline DYF404S1 & & & & & & $\checkmark$ \\
\hline
\end{tabular}

a Promega

b Thermo Fisher Scientific

c Non-commercial

Yfiler Y-STR profiles. The special force team ordered the analysis of additional 38 Y-STR markers to be performed by Ronny Decorte from the Department of Forensic Biomedical Sciences of the KU Leuven. Moreover, the NFI performed profiling of 13 Y-STRs known to have an untypically high mutation rate, so-called rapidly mutating (RM) Y-STRs. The scientific and investigative motivation behind this decision was based on the expectation that by increasing the number of Y-STRs, particularly using RM Y-STRs, the chance to detect Y-STR mutations that allow separating distant male relatives from close ones increases, which, in turn, decreases the suspect pool. Distant relatives identified because of observed mutations leading to non-matching extended Y-STR profiles could thus be excluded from being relevant to the case, whereas close relatives with matching extended Y-STR profiles 
provided focused leads in the search for the unknown perpetrator.

However, to the surprise of everybody in the team, it turned out that one of the volunteers with a Y-STR profile match also showed an autosomal STR profile match with the semen trace. This finding provided strong evidence that this particular man was the donor of the semen trace. Soon after his subsequent arrest, Jasper S. of Dutch European ancestry and from Oudwoude located $2.5 \mathrm{~km}$ away from the murder site confessed that he had raped and murdered Marianna Vaatstra during the night of April 30, 1999. As a result, he was found guilty by the court in Leeuwarden and sentenced for 18 years in prison on April 2013, 14 years after the murder. Because the power of the applied Y-STR dragnet for familial searching has widely been communicated, he likely expected that several of his close and/or extended family members from the region would participate in the DNA dragnet, and would, therefore, reveal his identity eventually. He may have thought that direct participation was his only chance to escape, by hoping that the authorities, having to collect thousands of DNA profiles for the first time in Dutch history, would eventually make mistakes. This could explain why he did not show-up for voluntary sampling at his designated place during the first days of sampling, but only participated in the last days of voluntary sample collection at a different collection place. In the end, Jasper S. was identified as the murderer of Marianne Vaatstra, because he directly participated in the DNA dragnet. Obviously, under the scenario of direct participation, his DNA identification would also have occurred when the conventional autosomal STRs had been used instead of Y-STRs. However, it remains unclear whether he would, indeed, have voluntarily participated in an autosomal STR dragnet; the increased power of relative identification with the Y-STR dragnet had widely been communicated. In any case, it can be expected that the combined approach of a dense Y-STR dragnet, genealogy investigation, and additional Y-STR testing, particularly the use of RM Y-STRs, would have allowed the special force team to trace him eventually, even if he had not participated in the Y-STR dragnet.

This case particularly demonstrates the necessity and suitability of forensic Y-chromosome DNA analysis, which is discussed in more detail in the following chapters. One aspect not being further outlined below is the routine forensic use of Y-chromosome DNA for inferring the biological sex of a trace donor. In brief, biological sex information can be inferred from DNA via analysing genetic loci located on both sex chromosomes. In addition to STRs, all currently used commercial autosomal STR profiling kits target a small fragment of the amelogenin gene, which has a length polymorphism between its X-chromosome and Y-chromosome copies that is detected in the analysis. However, the use of amelogenin as sole sex marker in forensic DNA testing has repeatedly been criticized (Brinkmann 2002; Santos et al. 1998; Steinlechner et al. 2002; Thangaraj et al. 2002) due to rarely occurring Y-chromosome deletions that include the amelogenin locus, which makes such males to appear as female instead in the test outcomes. Nevertheless, until now, amelogenin remains the only sex marker in current commercial DNA profiling kits, but considerations should be given to including more sex-indicating DNA markers in the future.

\section{Y-STRs for paternal lineage identification}

Standard DNA profiling using sets of well-selected, largely standardized, highly polymorphic autosomal STRs, is very suitable for identifying a donor of a single-source crime scene trace, as long as this person's STR profile is already known to the investigating authorities. Nowadays, such knowledge typically comes from forensic DNA databases, where STR profiles of convicted crime offenders are stored and STR profiles obtained from crime scene traces are compared with to look for a match. Obviously, this comparative autosomal STR profile matching for human identification is not successful for completely unknown perpetrators, whose STR profiles are not yet available. Moreover, autosomal STR profiling is compromised in cases where more than one person has contributed to a crime scene trace (multiple-source samples). Only under certain favourable circumstances, such as one donor contributing much more DNA to the mixed stain than the other(s), it is possible to single out complete autosomal STR profiles from such mixed stains, while in many such cases, it is not. There is one type of crime cases, where multiple-source material typically comes from male and female contributors, and the to-be-identified male usually is the minor contributor. This is cases of sexual assault, where DNA analysis needs to be performed on vaginal swabs to identify the male rapist. In such cases, the autosomal STR profile of the female major contributor from her excess of epithelial cells is known to the investigators from the victim's reference sample. Nevertheless, due to preferential PCR amplification of the major DNA component, and due to potential allele sharing between victim and perpetrator, it is often difficult, and in many cases impossible, to single out the autosomal STR profile of the male perpetrator from such mixed material. This is where Y-chromosome STR profiling comes into play, as only the male perpetrator, but not the female victim, carries a Y-chromosome.

Starting in 1992 with the publication of the first polymorphic STR discovered on the non-recombining part of the Y-chromosome (Roewer et al. 1992), and its immediate application to forensic casework (Roewer and Epplen 
1992), more and more Y-STR markers were subsequently developed for forensic Y-STR haplotyping (Gopinath et al. 2016; Hall and Ballantyne 2003; Hanson and Ballantyne 2004, 2007; Kayser et al. 1997; Krenke et al. 2005; Lim et al. 2007; Mulero et al. 2006; Rodig et al. 2008; Thompson et al. 2013; Vermeulen et al. 2009). Up to 27 markers are currently included in commercial Y-STR kits [Yfiler Plus, Thermo Fisher Scientific (Gopinath et al. 2016)] (Table 1). Due to the achieved high haplotype diversity, these tools allow for the characterization of a paternal lineage with high, albeit not maximal, degree of certainty, especially when the tested sample donor comes from an outbred population (Purps et al. 2014; Vermeulen et al. 2009). Moreover, these commercial Y-STR kits allow the detection and characterization of DNA from males in mixed stains with high excess of DNA from females, also in cases with very low quantities of DNA from the minor male contributor as typical in material from sexual assault (Purps et al. 2015). Recommendations on forensic analysis of Y-STRs have been established by the DNA Commission of the International Society of Forensic Genetics (Gill et al. 2001; Gusmao et al. 2006), and the Y-STR kits have forensically been validated (Gopinath et al. 2016; Krenke et al. 2005; Mulero et al. 2006; Thompson et al. 2013). This allows forensic practitioners not only to exclude male suspects from being involved in a crime via non-matching Y-STR haplotypes, but also to identify the paternal lineage that a trace donor belongs to via matching Y-STR haplotypes (Roewer 2009). For example, a recent study of hundreds of sexual assault cases, where Y-STR haplotyping had been applied together with standard autosomal STR profiling, showed that one tenth of these cases would have remained inconclusive without the use of Y-STRs, and furthermore, Y-STR haplotyping was three times more suitable to identify multiple male contributors than autosomal STR profiling (Purps et al. 2015).

Because of the completely linked inheritance of loci on the non-recombining part of the $\mathrm{Y}$ chromosome, the product rule of multiplying single locus allele frequencies cannot be applied, and, therefore, complete haplotype frequencies are needed for estimating Y-STR-based match probabilities. As Y-STR haplotypes are by magnitudes more variable than single autosomal STR loci, Y-STR haplotype reference databases must be by magnitudes larger than autosomal STR allele reference databases to provide reliable frequency estimates. The largest and most widely used Y-STR haplotype reference database is the YHRD (Willuweit and Roewer 2015), which currently (January 2017, Release 52) includes between 178,171 and 16,577 Y-STR haplotypes depending on the marker set. Searching a Y-STR haplotype obtained from a crime scene trace against the reference database provides the frequency of the haplotype needed for calculating the match probability.
As expected, a paternal lineage can be more accurately characterized via Y-STR haplotyping that the more Y-STR markers are considered. However, once a certain Y-STR set has been identified, as was the first set of nine markers referred to as Minimal Haplotype (Table 1) (Kayser et al. 1997), adding additional Y-STRs not necessarily improves paternal lineage resolution. Classically, population genetic studies are carried out to identify Y-STR markers suitable for paternal lineage identification based on diversity measures (Hanson and Ballantyne 2004, 2007; Kayser et al. 1997; Vermeulen et al. 2009). However, the general disadvantage of such diversity-driven approach is that the obtained Y-STR set is highly suitable for paternal lineage differentiation on some populations (i.e., the ones tested or those similar to the ones tested), but may not be in others (i.e., those with distant ancestry to the ones tested). For example, South African populations showed low levels of haplotype diversity with the 9 Y-STRs from the Minimal Haplotype (Leat et al. 2004), which could be improved drastically with 11 Y-STRs (all except one marker being different than $\mathrm{MH}$ ) selected from a South African population diversity data set of 45 Y-STRs (D'Amato et al. 2011).

However, some Y-STR markers seem more suitable than others to increase haplotype diversity and lineage resolution across populations. For instance, genotyping the 590 unrelated males from 51 worldwide populations included in the CEPH-HGDP panel for 67 Y-STRs, including the 17 standard Y-STRs from two commercial sets (PowerPlex-Y and Yfiler, see Table 1) and 49 non-standard Y-STRs (Lim et al. 2007), demonstrated that paternal lineage differentiation was increased over the commercial sets, globally and in all continental regions (except North Africa but represented by only a single population sample of small sample size) (Vermeulen et al. 2009). Six of the non-standard Y-STRs stood out in their value for lineages differentiation, in general, and for improving lineage differentiation when combined with standard Y-STRs (Vermeulen et al. 2009). Not surprisingly, these 6 Y-STRs were chosen by Promega (together with other markers already included in the Yfiler kit by Thermo Fisher Scientific) to expand and improve their PowerPlex-Y kit (Table 1), resulting in today's PowerPlex-Y23 kit (Thompson et al. 2013) (Table 1).

The general disadvantage of this diversity-driven approach for selecting useful Y-STRs can be overcome in part when using mutation rate estimates to select suitable markers. Although these two approaches are not entirely independent of one another, the mutation-driven approach only considers the actual genetic changes produced by mutations, while the diversity-driven approach additionally considers other factors such as migration, fluctuating population size, genetic drift, putative selection, etc., which can complicate the identification of suitable markers. However, the success of both approaches largely depends on 
study sample size. Even though all male-specific Y-STR loci are genetically linked, given the underlying mutational process of strand slippage during DNA replication, different Y-STR loci mutate independently from each other. The mutation rate of Y-STRs is mostly determined by the number of repeats, particularly the number of repeats in noninterrupted repetitive stretches, where more repeats lead to more DNA slippage during replication (Ballantyne et al. 2010; Kayser et al. 2004). Y-STRs with a higher mutation rate are expected to be generally more suitable for differentiating paternal lineage compared to those with a low mutation rate. For instance, this was seen in the aforementioned study (Vermeulen et al. 2009), where samples from various deep-rooted pedigrees were also analysed to get a preliminary indication of the mutation rates of the 49 nonstandard Y-STRs used. Notably, two of them (DYS570 and DYS576) stood out with much higher mutation rates compared to all other tested Y-STRs, including those from the commercial kits. These two were among the best 5 of all 67 tested Y-STRs, and among the best 6 of all 49 non-standard Y-STRs, eventually chosen for the PowerPlex-Y23 kit.

A later comprehensive mutation rate study of 186 Y-STRs in nearly 2000 DNA-confirmed father-son pairs not only confirmed the high mutation rate of these two Y-STRs, but identified 11 additional Y-STRs with similarly high mutation rates (i.e., a few mutations per 100 generations per each locus) (Ballantyne et al. 2010). These 13 RM Y-STRs (Table 1) are extremely useful for paternal lineage differentiation and identification (Ballantyne et al. 2012). For instance, in a large multicenter study including 12,272 unrelated males from 111 global populations, 12,156 (99\%) were differentiated by unique RM Y-STR haplotypes (Ballantyne et al. 2014). For comparison, 6975 (89.6\%) of a subset of 7784 unrelated men from 65 global population were separated with the Yfiler kit, while 7714 (99.1\%) were separated with the RM Y-STR set (Ballantyne et al. 2014). The value of RM Y-STRs to differentiate between close and distant male relatives will be discussed below.

Mutation rates of the same Y-STR loci can differ between populations; however, strong and thus practically relevant differences could only develop in populations that experienced an extreme bottleneck and founder effect followed by strong isolation. This is rare, perhaps, with the exception of remote island groups. Moreover, strong mutation rate differences would only occur if the founding males either predominantly carried Y-STR alleles with particularly long or with particularly short stretches of uninterrupted repeats favouring or disfavouring Y-STR mutations, respectively (Ballantyne et al. 2010). Such founder selection based on extremes in Y-STR repeat length is very unlikely to occur by chance or any other means. Although Y-STR mutation rate differences between and/ or within populations have been observed for the same loci (Goedbloed et al. 2009), they are rather small and can likely be explained by stochastic effects due to the rarity of occurring mutations given the small sample sizes used in some studies.

Overall, Y-STR haplotyping is very useful both for excluding suspects from involvement in a crime by demonstrating non-matching haplotypes, and for identifying groups of male relatives belonging to the same paternal lineage by demonstrating haplotype matches. However, commercial Y-STR kits are not suitable for male individual identification, because male relatives typically share the same resulting haplotype. Consequently, a match probability estimated for a Y-STR haplotype established with any of these kits not only applies to the tested suspect, but similarly to all of his untested male paternal relatives. It will then be up to the police to find out if, indeed, the matching suspect, or instead any of his close or distant male relatives, has left the trace at the crime scene. What is a disadvantage of Y-STRs for individual identification purposes serves as an advantage for paternity testing, other types of kinship testing, and for familial searching (as applied in the Vaatstra case).

\section{Y-STRs for paternity testing, kinship analysis, and familial searching}

Because Y-STR haplotypes are shared between paternally related men belonging to the same paternal lineage, Y-STR haplotyping is suitable for solving paternity disputes of male offspring, other types of paternal kinship questions, and for familial searching. It is also suitable to male identification cases involving human remains such as in disaster victim and missing person identification where only distant relatives are available. In paternity testing, Y-STR haplotyping is particularly suitable in deficiency cases, where the putative father of a male child is deceased and not available for DNA testing. With autosomal DNA profiling, the paternity of the unavailable putative father to the child can be established or rejected with the necessary high degree of certainty only if both parents of the deceased putative father are available for testing. If only one or none of the paternal grant parents of the male child are available, Y-STR profiling comes into play as long as any male relative of the deceased putative father is available for analysis. By use of standard Y-STRs with low-medium mutation rates [i.e., one or a few mutations every 1000 generations per each locus, (Goedbloed et al. 2009)], male relatives of the deceased putative father will share the same Y-STR haplotype with the putative father, and thus with his son, in case of biological paternity. Obviously, RM Y-STRs characterised by increased mutation rates are not suitable for paternity and kinship testing, as the mutations observed 
with increased probabilities will trouble the estimation of paternity/kinship probabilities.

As long as enough Y-STRs with low-medium mutation rates are analysed, allowing the clear characterization of the paternal lineage to which the putative father's paternal relative and the son belong, finding the same haplotype indicates biological paternity. The strength of probability of paternity will depend on the frequency of the Y-STR haplotype observed. The same applies in kinship analysis where the paternal relationship of one or more males is to be established or tested from hypotheses based on family record or archive information. However, even with such low-medium mutation rates, the chance of observing haplotypes that are different at certain Y-STRs due to rare mutations will generally increase the more Y-STRs are used. On the other hand, more Y-STRs can typically characterise and identify a paternal lineage better (see above), resulting in a dilemma in cases where haplotype differences are observed to decide between paternity/kinship with mutations versus non-paternity/non-kinship. For instance, in a Yfiler study using 1730 father-son pairs and finding a total of 84 mutations, one pair was found with mutations at 3 of the 17 Y-STRs, while two pairs with mutations at two Y-STRs, respectively (Goedbloed et al. 2009). Moreover, as it may be expected, when these father-son pairs were analysed for additional 169 Y-STRs, both the number of pairs with mutations at multiple Y-STRs, and the number of Y-STRs at which mutations were observed, increased (Ballantyne et al. 2010). In this extended study, 123 fatherson pairs were found with mutations at 3 Y-STRs, 42 pairs with mutations at 4 Y-STRs, and 3 pairs with mutations at 5, 6, 7, and 8 Y-STRs (Ballantyne et al. 2010). Therefore, instead of applying a fixed rule for excluding from paternity (or other kinship questions) based on exclusion constellations of the minimum of three Y-STRs, as argued previously (Kayser and Sajantila 2001), it is more sensible to use a flexible model. Such model shall consider the total number of Y-STRs analysed, their locus-specific mutation rate estimates, and the repeat number differences of the non-matching alleles observed. The latter is indicated, because the majority of Y-STR mutations represent single repeat changes (Ballantyne et al. 2010).

As long as the person in question is a male, the nonrecombining nature of male-specific Y-chromosome markers principally also allows to solve historical cases of paternity, or other types of paternal kinship dispute, as well as identification cases many generations after they occurred, which is impossible with recombining autosomal DNA. In historical identification cases, DNA from the remains of the historical man as well as from his living paternal relative assumed from family records must be available for Y-chromosome DNA analysis. In historical paternity cases, either DNA from the remains of the putative father and the son, or from living male descendent from both, as assumed from family records, must have available for Y-chromosome DNA analysis. When matching Y haplotypes are observed, true biological paternity/kinship or identification can be assumed, while different haplotypes indicate non-paternity or kinship. However, when observed haplotype differences are too many and/or too large in repeat number differences to be explainable by mutations, given the mutation rates of the Y-STRs and the number of separating meiosis in the family line, it is typically difficult, if not impossible, to find out at which male in the family line the non-biological paternity occurred. Moreover, matching haplotypes do not necessarily permit the conclusion of paternity or identity of the historical men, because his close relatives living at the time would likely have shared the same Y-chromosome haplotype, and, therefore, could have been the father/ wanted men with the same probability estimated from the Y haplotype.

Two examples of historical paternity and identification cases where Y-chromosome DNA was applied are mentioned here for illustration. In the paternity dispute of former US President Thomas Jefferson (1743-1826), Y-STR and Y-SNP analysis demonstrated that several currently living male relatives of Thomas Jefferson share the same $\mathrm{Y}$ haplotype as a living descendent of Eston Hemings Jefferson, son of Sally Hemings-the President's African American female slave (except for one repeat difference at one Y-STR, which could be easily explained by a mutation) (Foster et al. 1998). This indicates that President Jefferson had sired Eston Hemings Jefferson, or alternatively, his brother Randolph did; two scenarios such Y-chromosome analysis cannot differentiate. However, living male descendent of Thomas Corbin Woodson, the previously assumed full brother of Eston Hemings Jefferson, showed a very different $\mathrm{Y}$ haplotype, indicating that his biological father was a different man (Foster et al. 1998). In the identification case of King Richard III of England (1452-1485), various types of evidence including a complete match of the entire mitochondrial genome between the skeleton and an assumed living maternal relative of King Richard III gave a large likelihood that the skeleton is that of the King (King et al. 2014). However, the skeleton's Y haplotype did not match that of the King's living paternal relatives. Because of the overwhelming evidence in favour of identification, the $\mathrm{Y}$ discrepancy was concluded as indication of a false-paternity in any men of the extended family between the tested paternal relatives and the King (King et al. 2014).

The same principle of haplotype sharing between close and distant paternal male relatives as applied in paternity and kinship testing makes Y-STR haplotyping also suitable for familial searching, in forensic cases without autosomal DNA profile match (as applied in the Vaatstra case). However, different to paternity and other types of paternal 
kinship analysis, where only Y-STRs with low-medium mutation rates are suitable, Y-STR applications to familial searching may additionally require the use of RM Y-STRs (see also next chapter). This is in cases where haplotype matches based on low-medium mutating Y-STRs are seen with several persons (as in the Vaatstra case). Subsequently, RM Y-STRs need to be analysed, to separate-out the more distantly related male relatives identified by mutations, allowing to focus on the closely related ones highlighted by not showing mutations to guide the search for the unknown male perpetrator whose DNA was not available.

\section{Y-STRs for male relative differentiation towards male individual identification}

Due to the low-medium mutation rates of most of their Y-STRs, the commercial Y-STR kits have limitations in differentiating paternal lineage in inbred population, where the proportion of distantly related males is increased. Moreover, they typically fail to separate male relatives belonging to the same paternal lineage, thus not allowing individual identification, as is strongly desired in forensic DNA analysis in general. A way out of this dilemma was indicated by the first discovery of RM Y-STRs with untypically high mutation rates (Ballantyne et al. 2010). In principle, it can be expected that with sufficient numbers of RM Y-STR markers available, close, and especially distantly related men will be separated by means of observed mutations. Thus, individual identification can be achieved while maintaining the advantages of Y-chromosome DNA analysis for male-female mixed stain resolution.

Empirical evidence of male relative differentiation with the full 13 RM Y-STR set has steadily increased over the past few years. The discrimination rates currently mostsupported by available data are $27 \%$ for father-sons based on 2378 pairs (Ballantyne et al. 2014), 44\% for brothers and grandfather-grandsons separated by 2 meioses based on 480 pairs (Adnan et al. 2016), 55\% for cousins separated by three meiosis based on 308 pairs (Adnan et al. 2016), and $61 \%$ for male relatives separated by 4 meioses based on 277 pairs (Adnan et al. 2016). The most recent commercial Y-STR kits include two (PowerPlex-Y 23) and 6 (YfilerPlus) RM Y-STRs (Table 1). These kits, therefore, do not provide the full power of male relative differentiation as available with the complete set of 13 RM Y-STRs. Thus far, no commercial kit exists for all 13 RM Y-STRs, but non-commercial multiplex genotyping protocols are available (Alghafri et al. 2015; Ballantyne et al. 2012).

It is envisioned that if in a criminal case, a Y haplotype match is established with any of the commercial Y-STR kits, the full set of 13 RM Y-STRs shall be analysed to test whether the matching suspect, or his close or distant paternal male relatives, has left the trace at the crime scene. Furthermore, in constellations where there is a match of Y-STR haplotypes from commercial kits, and there is evidence that a close relative of the known suspect (such as a brother) may rather be the trace donor, reference DNA samples of both males shall be tested for the complete set of 13 RM Y-STRs. In case that a separating mutation at any of the RM Y-STRs is observed in any of the two reference DNA samples, the crime scene trace shall be analysed for RM Y-STRs, to establish to whom of the two men the evidence RM Y-STR haplotype matches. Although the current set of 13 RM Y-STRs has limitations in differentiating relatives, especially close ones (see numbers of male relative discrimination mentioned above), more RM Y-STRs may be identified in the future that will allow further increasing male relative differentiation rates, and may even eventually achieve individual identification of a man from Y-chromosome DNA analysis.

The ultra-high rate at which unrelated males can be differentiated with RM Y-STRs leads to the practical problem of putting statistical weight on a RM Y-STR haplotype match. Clearly, the more polymorphic a Y-STR haplotype is, the larger the haplotype reference database needs to be to deliver reliable frequency estimates. The problem of singletons in Y-STR reference databases has already been noticed for haplotypes established from commercial Y-STR kits. Such Y-STR haplotypes, however, are much less polymorphic than those generated from the full set of $13 \mathrm{RM}$ Y-STRs (Ballantyne et al. 2014), but the size of the YHRD is already large with currently (as of January 2017) 139,104 PowerPlex-Y haplotypes and 126,409 Yfiler haplotypes included (https://yhrd.org, Release 52). Despite this enormous size that could only be achieved via collaboration of the global forensic DNA community over decades, many Y-STR haplotypes obtained in routine forensic practise are not yet included in the YHRD. This poses a statistical problem on how to get reliable haplotype frequency estimates needed for calculating match probabilities. Forensic statisticians have been trying to develop solutions (Andersen et al. 2013; Brenner 2010; Buckleton et al. 2011), but no consensus on the most suitable method has been reached thus far. Clearly, this problem becomes more severe for haplotypes based on RM Y-STRs that are much more variable than those obtained from commercial Y-STR kits (Ballantyne et al. 2014), including the most recent kits (Purps et al. 2014).

\section{The $Y$ chromosome for inferring paternal bio-geographic ancestry}

As mentioned above in the context of the Vaatstra case, the $\mathrm{Y}$ chromosome is highly suitable to provide information 
about the geographic region a person's paternal ancestors originate from, i.e., bio-geographic ancestry. Forensic DNA testing for bio-geographic ancestry is useful in cases where autosomal STR profile matches are lacking, because the perpetrator is completely unknown to the investigators. In such cases, bio-geographic ancestry information obtained from evidence DNA [at best in combination with information regarding externally visible characteristics and age (Kayser 2015)] can guide police investigations towards finding unknown perpetrators (Phillips 2015). Similarly, DNA testing on bio-geographic ancestry can be useful in missing person cases, including disaster victim identification cases, without any knowledge about the possible identity of the person to whom the biological remains belong.

In general, the suitability of Y-chromosome DNA for inferring paternal bio-geographic ancestry comes from its escape from recombination, as it is also seen for maternal ancestry with maternally inherited mitochondrial $(\mathrm{mt})$ DNA. Under the absence of recombination, once a mutation has occurred, it is not removed from the gene pool, unless no male (or male and female in case of mtDNA) offspring exists. Both uniparentally inherited parts of the human genome ( $\mathrm{Y}$ and $\mathrm{mt})$ are, therefore, more prone to genetic drift, which can produce genetic differences between geographic regions simply by chance. Further contributing to the suitability of the Y-chromosome for ancestry inference is certain elements of human culture, such as patrilocal residence and polygyny, which increase Y-chromosome differences over geographic distance. For decades, Y-chromosomal DNA polymorphisms were explored to trace bio-geographic ancestry of individuals and populations, in the beginning mostly from an evolutionary perspective to understand population origins and migration history worldwide (Underhill and Kivisild 2007). Such research produced a wealth of knowledge on the geographic distribution of Y-chromosome genetic diversity, which serves as the basis for the forensic applications of paternal bio-geographic ancestry inference, particularly for Y-SNPs.

Because of their about 100,000 lower mutation rates relative to most Y-STRs (Ballantyne et al. 2010; Xue et al. 2009), geographic ancestry signatures are kept much longer at Y-SNPs before being diluted via mutations, relative to Y-STRs. Therefore, Y-SNPs are generally more suitable for paternal bio-geographic ancestry inference than Y-STRs. It is widely assumed that modern humans go back to a single recent common origin in Africa, that they first left Africa about 100,000 years, and arrive in the different continental regions between 60,000 and 15,000 years ago, depending on the region. This history equals enough generation steps to allow Y-chromosome mutations generating continental differences at various Y-SNPs. Furthermore, subsequent population movements, male-driven cultural traits, genetic drift, and various other factors have produced Y-SNP frequency differences between geographic regions and, albeit less pronounced, between subregions.

In recent years, more and more large-scale resequencing studies using massively parallel sequencing (MPS) technologies, also referred to as next-generation sequencing (NGS), have produced a large number of newly discovered Y-SNPs (Batini et al. 2015; Francalacci et al. 2013; Hallast et al. 2015; Scozzari et al. 2014; Trombetta et al. 2015), much larger than previously found with other technologies (Karafet et al. 2008). They are placed into their phylogenetic position via routinely updated global Y-chromosome trees made available as open resource by the International Society of Genetic Genealogy (ISOGG) (http://isogg.org/ tree/index.html). A minimal reference phylogeny for the human Y-chromosome, representing an abbreviated version of the Y-tree showing only the principal branches together with the geographic regions of predominant occurrence, is available via Phylotree-Y (http://www.phylotree.org/Y/ tree/index.htm) (van Oven et al. 2014). For orientation, Table 2 provides a selected list of Y-SNP haplogroups with their geographic regions of predominant occurrence that are informative for paternal bio-geographic ancestry inference. Moreover, various Y-SNP genotyping tools suitable to low-quantity and low-quality DNA have been developed for forensic and other applications (such as anthropology and genealogy) (Brion et al. 2005; Gomes et al. 2010; van Oven et al. 2011, 2012, 2013). Due to the SNaPshot technology used, these tools have restrictions in the number of Y-SNPs analysed simultaneously, providing limitation on the geographic resolution at which paternal bio-geographic ancestry can be obtained with such tools. Many Y-SNPs together with their respective genotyping tools are available that allow paternal bio-geographic ancestry inference on the level of continental resolution. For some continental regions, such as Europe, Y-SNPs also allow subregional inference of paternal ancestry (Balaresque et al. 2010; Batini et al. 2015; Cruciani et al. 2011). However, for many of the recently discovered and already phylogenetically mapped Y-SNPs, population data are lacking, so that their suitability for paternal bio-geographic ancestry testing needs to be established in the future via the generation of population data to reveal their geographic distributions.

Some Y-SNP-based haplogroups with strong frequency differences between geographic (sub)regions display a strong-enough correlation with their associated Y-STR haplotype diversity, so that the geographic regions indicated by the Y-SNP haplogroup can also be inferred from associated Y-STR haplotypes (as performed in the Vaatstra case). To cover more of the geographic information of a Y-STR haplotype, a nearest neighbour Y-STR haplotype search in the reference database can help, as this would take mutation steps into account. Well-known examples are the major haplogroups R1b indicating Western European paternal 
Table 2 Selective list of Y-SNP-based haplogroups informative for paternal bio-geographic ancestry inference worldwide

\begin{tabular}{|c|c|c|c|}
\hline Haplogroup & Defining bi-allelic marker(s) & Respective rs number(s) & Major geographic area(s) of occurrence \\
\hline A00-L1086 & L1086; L1159; L1284 & N/A; N/A; N/A & Central Africa \\
\hline A0-V148 & V148; V166; L896; L991 & $\begin{array}{l}\text { rs181335666; rs187287389; N/A; } \\
\text { N/A }\end{array}$ & Central Africa, West Africa \\
\hline A1-M31 & M31; P82; V4 & rs369315948; N/A; rs187409543 & West Africa, North Africa \\
\hline A2-V50 & V50; L602 & rs189205028; rs576471146 & Southern Africa, Central Africa \\
\hline A3-M32 & M32 & rs558241924 & East Africa, Southern Africa \\
\hline B-M60 & M60; M181; V244 & rs2032623; rs2032599; rs112298449 & Central Africa, Southern Africa, East Africa \\
\hline D-M174 & M174; CTS94; JST021355 & rs2032602; rs199881488; rs2267802 & East Asia \\
\hline E-M96 & M96; M40; P29 & rs9306841; rs9786608; rs60115999 & Africa, West Asia, Southern Europe \\
\hline E-V13 & V13; V36 & rs368031074; rs371443469 & Southern Europe \\
\hline E-M293 & M293 & rs9341316 & Southern Africa \\
\hline C-M130 & M130; M216 & rs35284970; rs2032666 & $\begin{array}{l}\text { Central Asia, Northern Asia, North America, East Asia, } \\
\text { Southeast Asia, Wallacea, Near Oceania, Remote } \\
\text { Oceania, Australia }\end{array}$ \\
\hline C-M8 & M8; M105 & rs3899; rs2032612 & Japan \\
\hline $\mathrm{C}-\mathrm{V} 20$ & $\mathrm{~V} 20$ & rs182352067 & Southern Europe \\
\hline C-M356 & M356 & N/A & South Asia, Central Asia \\
\hline C-B65 & B65 & rs374541802 & Indonesia, Philippines \\
\hline C-M38 & M38 & rs369611932 & Wallacea, Near Oceania, Remote Oceania \\
\hline C-M208 & M208 & rs 2032659 & Near Oceania, Remote Oceania \\
\hline C-P33 & P33 & N/A & Remote Oceania \\
\hline C-PH41 & $\begin{array}{l}\text { PH41; PH338; PH4186; } \\
\text { PH4682 }\end{array}$ & N/A; N/A; N/A; N/A & Australia \\
\hline C-M217 & M217; P44; Z1453 & rs2032668; N/A; N/A & Central Asia, Northeast Asia, Northern Americas \\
\hline C-P39 & P39 & N/A & Northern Americas \\
\hline G-M201 & M201; P257 & rs2032636; rs2740980 & West Asia, Europe, Central Asia \\
\hline H-L901 & L901; M3035 & rs567848586; rs74378870 & South Asia \\
\hline I-M170 & M170; M258; U179 & rs2032597; rs9341301; rs2319818 & Europe, West Asia \\
\hline J-M304 & M304; P209 & rs13447352; rs17315835 & $\begin{array}{l}\text { West Asia, North Africa, Horn of Africa, Southern } \\
\text { Europe, Central Asia, South Asia }\end{array}$ \\
\hline L-M20 & M20 & rs3911 & South Asia, West Asia \\
\hline M-P397 & P397; P399; PR2099 & N/A; N/A; rs369017623 & Wallacea, Near Oceania, Remote Oceania, Australia \\
\hline M-P34 & P34 & N/A & Near Oceania \\
\hline M-M10072 & M10072; FGC38729; Z33118 & rs566812523; N/A; rs368850080 & Australia \\
\hline N-M231 & M231 & rs9341278 & Northern Asia, Northern Europe \\
\hline O-M175 & M175; P186 & rs2032678 & East Asia, Southeast Asia, Remote Oceania \\
\hline Q-M242 & M242 & rs8179021 & Northern Asia, Central Asia, Americas \\
\hline Q-M3 & M3 & rs3894 & Americas \\
\hline Q-Z780 & $\mathrm{Z780}$ & N/A & Americas \\
\hline R-M207 & M207 & rs2032658 & $\begin{array}{l}\text { Europe, West Asia, Central Asia, South Asia, North } \\
\text { Africa, Central Africa }\end{array}$ \\
\hline R-M458 & M458 & rs375323198 & Eastern Europe, Caucasus region \\
\hline R-Z284 & $\mathrm{Z} 284$ & N/A & Northwest Europe \\
\hline R-Z93 & Z93 & rs566323605 & South Asia, Central Asia \\
\hline $\mathrm{R}-\mathrm{V} 88$ & V88 & rs180946844 & Africa \\
\hline R-M412 & M412 & rs9786140 & Western Europe \\
\hline R-M479 & M479 & rs 372157627 & South Asia, Central Asia \\
\hline S-M254 & M254 & rs9341297 & Near Oceania \\
\hline T-M184 & M184 & rs 20320 & $\begin{array}{l}\text { West Asia, Horn of Africa, North Africa, Southern } \\
\text { Europe, South Asia }\end{array}$ \\
\hline
\end{tabular}


ancestry and R1a indicating Eastern European paternal ancestry. However, not many Y-STR haplotypes with strong geographic signatures are known. Notably, the YHRD has recently been expanded to Y-SNP data and currently (as of January 2017, Release 52) includes 20,187 Y-SNP profiles (https://yhrd.org).

\section{The future of forensic Y-chromosome DNA analysis}

In recent years, commercial Y-STR kits have seen an improvement in the number of Y-STR markers included, enabling increased paternal lineage resolution. However, these kits cannot differentiate all unrelated men in a population, nor do they allow the discrimination of related men. It, therefore, is envisioned that future commercial Y-STR kits will include more markers, particularly more RM Y-STRs. However, including more RM Y-STRs will lead to a further increasing problem of putting a statistical weight on an observed haplotype match because of the ultra-high diversity of RM Y-STR haplotypes requiring extremely large reference databases for estimating a somewhat reliable frequency of the matching haplotype needed for estimating the match probability, unless new statistical solutions will be developed.

Moreover, the two different forensic applications of Y-STR haplotyping, on one hand paternal lineage identification, for which Y-STRs with high mutation rates are most suitable, and on the other hand paternal/kinship determination and familial searching, for which Y-STRs with lowmedium mutation rates are most suitable, require that both types of Y-STR markers need to be considered in adequate numbers in future commercial kits. However, including many more than 27 Y-STRs, as is currently the maximum in commercial Y-STR kits, is approaching the limits of fluorescence-based fragment length analysis with capillary electrophoresis (CE), which represents the method of choice for routine forensic Y-STR analysis. This can be overcome with more fluorescence dyes being employed, requiring new chemistry and new instrumentation being developed. Alternatively, separate Y-STR kits for the two forensic applications could be developed, which would at least reduce the CE-based multiplexing problem by half. Targeted MPS technologies may serve as an alternative for multiplexing large numbers of Y-STRs, as already applied for autosomal STRs, Y-STRs, and SNPs with the ForenSeq kit (Illumina). However, the current MPS platforms suitable for forensic DNA analysis come with strong size limitations on the sequenced fragment length. Although the obtained short sequencing reads are suitable for many Y-STRs with low-medium mutation rates, they are not sufficient for RM Y-STRs with long repetitive stretches. Hence, MPS technologies enabling the sequencing of longer fragments from low-quality/quantity forensic DNA will have to be developed.

Regarding forensic ancestry testing using Y-chromosome markers, future work needs to provide more knowledge about the geographic distribution of many of the recently discovered Y-SNPs, to establish how useful they are for improving the geographic resolution of paternal ancestry inference. It is expected that such knowledge will allow paternal bio-geographic ancestry inference to be moved from the current level of mostly continental resolution to a much more detailed geographic resolution. As with Y-STRs, also for Y-SNPs, the limitation in multiplexing capacity of the genotyping technologies currently used in forensic DNA analysis has to be overcome, to take full advantage of the large number of Y-SNPs needed for inferring bio-geographic ancestry on a detailed level. Here, current targeted MPS technologies are highly promising because of their large multiplex capacity together with their short sequencing reads, given the single base pair nature of Y-SNPs. For example, a recent proof-of-principle study demonstrated that 530 Y-SNPs can be analysed simultaneously in a single targeted MPS run (Ralf et al. 2015). In any case, to achieve accurate bio-geographic ancestry inference of a person from its DNA, ancestry-informative SNPs from the $\mathrm{Y}$ chromosome for paternal ancestry inference need to be combined with those from mitochondrial (mt) DNA for maternal ancestry inference and from autosomal DNA for bi-parental ancestry inference. Such a combined genetic approach will allow inferring bio-geographic ancestry of admixed persons whose biological ancestors come from very different geographic regions, which is impossible with Y-chromosome DNA (or mt DNA) alone.

Finally, some authors, including in this special journal issue (Calafell and Larmuseau 2016), have suggested additional forensic applications of the human Y-chromosome than discussed above, for instance predicting a man's surname from his Y-chromosome DNA (Calafell and Larmuseau 2016; King and Jobling 2009). In patrilineal societies, which most human societies are, surnames are transferred from the father to all his offspring, as is the human Y chromosome to his sons. In such societies, co-ancestry of surnames and Y-chromosomes is, therefore, expected. However, this comes under the prerequisites that a surname was not given to/taken by unrelated men, and that the rate of non-biological paternity is very small, at best zero, since the surname started to be used. In reality, many surnames have been given multiple times to unrelated men, so that men from different paternal families with different Y-chromosomes can have the same surname. The co-ancestry of surnames and Y-chromosomes is additionally decreased with any non-biological paternity occurring (around $1-2 \%$ ), and through adoptions from outside the paternal family. So far, data evidence is limited and restricted to 
mainly three European countries, i.e., Great Britain (King et al. 2006), Ireland (McEvoy and Bradley 2006), and Spain (Martinez-Cadenas et al. 2016). Although strong coancestry has been observed for some rare surnames, common ones showed low or no Y-chromosome correlation (King et al. 2006; Martinez-Cadenas et al. 2016), which limits broad forensic applications. Nevertheless, the combined use of Y-chromosome data and surname information can be highly valuable in specific forensic cases, for instance when Y-chromosome-based familial searching is combined with genealogy investigation, as successfully applied for solving the murder case of Marianne Vaatstra.

Acknowledgements The author thanks the current and former members of the Department of Genetic Identification of Erasmus MC University Medical Center Rotterdam (formerly known as Department of Forensic Molecular Biology), who were involved in Y-chromosome research and applications, particularly Kaye Ballantyne, Miriam Goedbloed, Mannis van Oven, Arwin Ralf, Lakshmi Chaitanya, Silke Brauer, Mark Vermeulen, Victoria Keerl, Ying Choi, Oscar Lao, and Andreas Wollstein. He is grateful to Ron Rintjema, Arnoud Kal, and Peter de Knijff for their useful information and comments on the Vaatstra case investigation, and Mannis van Oven for help with Table 2. Athina Vidaki is acknowledged for her useful comments helping to improve the final manuscript. Finally, the author expresses his deep appreciation towards colleagues from all sides (academia, service, and industry), whose contributions helped initiating, establishing, and further improving forensic Y-chromosome DNA analysis over the last 25 years. The author's original work on fundamental and applied aspects of Y-chromosome genetics is supported by Erasmus MC University Medical Center Rotterdam (http://www.erasmusmc. $\mathrm{nl}$ ), and was previously funded by The Netherlands Genomics Initiative/Netherlands Organization for Scientific Research (NWO) within the framework of the Forensic Genomics Consortium Netherlands (FGCN) (http://www.forensicgenomics.nl) and by The Netherlands Forensic Institute (NFI) (http://www.forensicinstitute.nl).

\section{Compliance with ethical standards}

Conflict of interest The author declares that he has no conflict of interest.

Open Access This article is distributed under the terms of the Creative Commons Attribution 4.0 International License (http://creativecommons.org/licenses/by/4.0/), which permits unrestricted use, distribution, and reproduction in any medium, provided you give appropriate credit to the original author(s) and the source, provide a link to the Creative Commons license, and indicate if changes were made.

\section{References}

Adnan A, Ralf A, Rakha A, Kousouri N, Kayser M (2016) Improving empirical evidence on differentiating closely related men with RM Y-STRs: a comprehensive pedigree study from Pakistan. Forensic Sci Int Genet 25:45-51

Alghafri R, Goodwin W, Ralf A, Kayser M, Hadi S (2015) A novel multiplex assay for simultaneously analysing 13 rapidly mutating Y-STRs. Forensic Sci Int Genet 17:91-98
Andersen MM, Caliebe A, Jochens A, Willuweit S, Krawczak M (2013) Estimating trace-suspect match probabilities for singleton Y-STR haplotypes using coalescent theory. Forensic Sci Int Genet 7:264-271

Balaresque P, Bowden GR, Adams SM, Leung HY, King TE, Rosser ZH, Goodwin J, Moisan JP, Richard C, Millward A, Demaine AG, Barbujani G, Previdere C, Wilson IJ, Tyler-Smith C, Jobling MA (2010) A predominantly neolithic origin for European paternal lineages. PLoS Biol 8:e1000285

Ballantyne KN, Goedbloed M, Fang R, Schaap O, Lao O, Wollstein A, Choi Y, van Duijn K, Vermeulen M, Brauer S, Decorte R, Poetsch M, von Wurmb-Schwark N, de Knijff P, Labuda D, Vezina H, Knoblauch H, Lessig R, Roewer L, Ploski R, Dobosz T, Henke L, Henke J, Furtado MR, Kayser M (2010) Mutability of Y-chromosomal microsatellites: rates, characteristics, molecular bases, and forensic implications. Am J Hum Genet $87: 341-353$

Ballantyne KN, Keerl V, Wollstein A, Choi Y, Zuniga SB, Ralf A, Vermeulen M, de Knijff P, Kayser M (2012) A new future of forensic Y-chromosome analysis: rapidly mutating Y-STRs for differentiating male relatives and paternal lineages. Forensic Sci Int Genet 6:208-218

Ballantyne KN, Ralf A, Aboukhalid R, Achakzai NM, Anjos MJ, Ayub Q, Balazic J, Ballantyne J, Ballard DJ, Berger B, Bobillo C, Bouabdellah M, Burri H, Capal T, Caratti S, Cardenas J, Cartault F, Carvalho EF, Carvalho M, Cheng B, Coble MD, Comas D, Corach D, D'Amato ME, Davison S, de Knijff P, De Ungria MC, Decorte R, Dobosz T, Dupuy BM, Elmrghni S, Gliwinski M, Gomes SC, Grol L, Haas C, Hanson E, Henke J, Henke L, Herrera-Rodriguez F, Hill CR, Holmlund G, Honda K, Immel UD, Inokuchi S, Jobling MA, Kaddura M, Kim JS, Kim SH, Kim W, King TE, Klausriegler E, Kling D, Kovacevic L, Kovatsi L, Krajewski P, Kravchenko S, Larmuseau MH, Lee EY, Lessig R, Livshits LA, Marjanovic D, Minarik M, Mizuno N, Moreira H, Morling N, Mukherjee M, Munier P, Nagaraju J, Neuhuber F, Nie S, Nilasitsataporn P, Nishi T, Oh HH, Olofsson J, Onofri V, Palo JU, Pamjav H, Parson W, Petlach M, Phillips C, Ploski R, Prasad SP, Primorac D, Purnomo GA, Purps J, Rangel-Villalobos H, Rebala K, Rerkamnuaychoke B, Gonzalez DR, Robino C, Roewer L, Rosa A, Sajantila A, Sala A, Salvador JM, Sanz P, Schmitt C, Sharma AK, Silva DA, Shin KJ et al (2014) Toward male individualization with rapidly mutating y-chromosomal short tandem repeats. Hum Mutat 35:1021-1032

Batini C, Hallast P, Zadik D, Delser PM, Benazzo A, Ghirotto S, Arroyo-Pardo E, Cavalleri GL, de Knijff P, Dupuy BM, Eriksen HA, King TE, Lopez de Munain A, Lopez-Parra AM, Loutradis A, Milasin J, Novelletto A, Pamjav H, Sajantila A, Tolun A, Winney B, Jobling MA (2015) Large-scale recent expansion of European patrilineages shown by population resequencing. Nat Commun 6:7152

Brenner CH (2010) Fundamental problem of forensic mathematicsthe evidential value of a rare haplotype. Forensic Sci Int Genet 4:281-291

Brinkmann B (2002) Is the amelogenin sex test valid? Int J Leg Med 116:63

Brion M, Sobrino B, Blanco-Verea A, Lareu MV, Carracedo A (2005) Hierarchical analysis of 30 Y-chromosome SNPs in European populations. Int J Leg Med 119:10-15

Buckleton JS, Krawczak M, Weir BS (2011) The interpretation of lineage markers in forensic DNA testing. Forensic Sci Int Genet 5:78-83

Calafell F, Larmuseau MHD (2016) The Y chromosome as the most popular marker in genetic genealogy benefits interdisciplinary research. Hum Genet. doi:10.1007/s00439-016-1740-0

Cruciani F, Trombetta B, Antonelli C, Pascone R, Valesini G, Scalzi V, Vona G, Melegh B, Zagradisnik B, Assum G, Efremov GD, 
Sellitto D, Scozzari R (2011) Strong intra- and inter-continental differentiation revealed by $\mathrm{Y}$ chromosome SNPs M269, U106 and U152. Forensic Sci Int Genet 5:e49-e52

D'Amato ME, Bajic VB, Davison S (2011) Design and validation of a highly discriminatory 10-locus Y-chromosome STR multiplex system. Forensic Sci Int Genet 5:122-125

de Knijff P, Kayser M, Caglia A, Corach D, Fretwell N, Gehrig C, Graziosi G, Heidorn F, Herrmann S, Herzog B, Hidding M, Honda K, Jobling M, Krawczak M, Leim K, Meuser S, Meyer E, Oesterreich W, Pandya A, Parson W, Penacino G, PerezLezaun A, Piccinini A, Prinz M, Roewer L et al (1997) Chromosome Y microsatellites: population genetic and evolutionary aspects. Int J Leg Med 110:134-149

Foster EA, Jobling MA, Taylor PG, Donnelly P, de Knijff P, Mieremet R, Zerjal T, Tyler-Smith C (1998) Jefferson fathered slave's last child. Nature 396:27-28

Francalacci P, Morelli L, Angius A, Berutti R, Reinier F, Atzeni R, Pilu R, Busonero F, Maschio A, Zara I, Sanna D, Useli A, Urru MF, Marcelli M, Cusano R, Oppo M, Zoledziewska M, Pitzalis M, Deidda F, Porcu E, Poddie F, Kang HM, Lyons R, Tarrier B, Gresham JB, Li B, Tofanelli S, Alonso S, Dei M, Lai S, Mulas A, Whalen MB, Uzzau S, Jones C, Schlessinger D, Abecasis GR, Sanna S, Sidore C, Cucca F (2013) Low-pass DNA sequencing of 1200 Sardinians reconstructs European Y-chromosome phylogeny. Science 341:565-569

Gill P, Brenner C, Brinkmann B, Budowle B, Carracedo A, Jobling MA, de Knijff P, Kayser M, Krawczak M, Mayr WR, Morling N, Olaisen B, Pascali V, Prinz M, Roewer L, Schneider PM, Sajantila A, Tyler-Smith C (2001) DNA Commission of the International Society of Forensic Genetics: recommendations on forensic analysis using Y-chromosome STRs. Forensic Sci Int 124:5-10

Goedbloed M, Vermeulen M, Fang RN, Lembring M, Wollstein A, Ballantyne K, Lao O, Brauer S, Kruger C, Roewer L, Lessig R, Ploski R, Dobosz T, Henke L, Henke J, Furtado MR, Kayser M (2009) Comprehensive mutation analysis of 17 Y-chromosomal short tandem repeat polymorphisms included in the AmpFISTR Yfiler PCR amplification kit. Int J Leg Med 123:471-482

Gomes V, Sanchez-Diz P, Amorim A, Carracedo A, Gusmao L (2010) Digging deeper into East African human Y chromosome lineages. Hum Genet 127:603-613

Gopinath S, Zhong C, Nguyen V, Ge J, Lagace RE, Short ML, Mulero JJ (2016) Developmental validation of the Yfiler((R)) Plus PCR amplification kit: an enhanced Y-STR multiplex for casework and database applications. Forensic Sci Int Genet 24:164-175

Gusmao L, Butler JM, Carracedo A, Gill P, Kayser M, Mayr WR, Morling N, Prinz M, Roewer L, Tyler-Smith C, Schneider PM (2006) DNA Commission of the International Society of Forensic Genetics (ISFG): an update of the recommendations on the use of Y-STRs in forensic analysis. Forensic Sci Int 157:187-197

Hall A, Ballantyne J (2003) The development of an 18-locus Y-STR system for forensic casework. Anal Bioanal Chem 376:1234-1246

Hallast P, Batini C, Zadik D, Maisano Delser P, Wetton JH, ArroyoPardo E, Cavalleri GL, de Knijff P, Destro Bisol G, Dupuy BM, Eriksen HA, Jorde LB, King TE, Larmuseau MH, Lopez de Munain A, Lopez-Parra AM, Loutradis A, Milasin J, Novelletto A, Pamjav H, Sajantila A, Schempp W, Sears M, Tolun A, Tyler-Smith C, Van Geystelen A, Watkins S, Winney B, Jobling MA (2015) The Y-chromosome tree bursts into leaf: 13,000 high-confidence SNPs covering the majority of known clades. Mol Biol Evol 32:661-673
Hanson EK, Ballantyne J (2004) A highly discriminating 21 locus Y-STR "megaplex" system designed to augment the minimal haplotype loci for forensic casework. J Forensic Sci 49:40-51

Hanson EK, Ballantyne J (2007) An ultra-high discrimination y chromosome short tandem repeat multiplex DNA typing system. PLoS One 2:e688

Karafet TM, Mendez FL, Meilerman MB, Underhill PA, Zegura SL, Hammer MF (2008) New binary polymorphisms reshape and increase resolution of the human Y chromosomal haplogroup tree. Genome Res 18:830-838

Kayser M (2015) Forensic DNA phenotyping: predicting human appearance from crime scene material for investigative purposes. Forensic Sci Int Genet 18:33-48

Kayser M, Sajantila A (2001) Mutations at Y-STR loci: implications for paternity testing and forensic analysis. Forensic Sci Int 118:116-121

Kayser M, Caglia A, Corach D, Fretwell N, Gehrig C, Graziosi G, Heidorn F, Herrmann S, Herzog B, Hidding M, Honda K, Jobling M, Krawczak M, Leim K, Meuser S, Meyer E, Oesterreich W, Pandya A, Parson W, Penacino G, Perez-Lezaun A, Piccinini A, Prinz M, Schmitt C, Roewer L et al (1997) Evaluation of Y-chromosomal STRs: a multicenter study. Int J Leg Med 110(125-33):141-149

Kayser M, Kittler R, Erler A, Hedman M, Lee AC, Mohyuddin A, Mehdi SQ, Rosser Z, Stoneking M, Jobling MA, Sajantila A, Tyler-Smith C (2004) A comprehensive survey of human Y-chromosomal microsatellites. Am J Hum Genet 74:1183-1197

King TE, Jobling MA (2009) What's in a name? Y chromosomes, surnames and the genetic genealogy revolution. Trends Genet 25:351-360

King TE, Ballereau SJ, Schurer KE, Jobling MA (2006) Genetic signatures of coancestry within surnames. Curr Biol 16:384-388

King TE, Fortes GG, Balaresque P, Thomas MG, Balding D, Delser PM, Neumann R, Parson W, Knapp M, Walsh S, Tonasso L, Holt J, Kayser M, Appleby J, Forster P, Ekserdjian D, Hofreiter M, Schurer K (2014) Identification of the remains of King Richard III. Nat Commun 5:5631

Krenke BE, Viculis L, Richard ML, Prinz M, Milne SC, Ladd C, Gross AM, Gornall T, Frappier JR, Eisenberg AJ, Barna C, Aranda XG, Adamowicz MS, Budowle B (2005) Validation of male-specific, 12-locus fluorescent short tandem repeat (STR) multiplex. Forensic Sci Int 151:111-124

Leat N, Benjeddou M, Davison S (2004) Nine-locus Y-chromosome STR profiling of Caucasian and Xhosa populations from Cape Town, South Africa. Forensic Sci Int 144:73-75

Lim SK, Xue Y, Parkin EJ, Tyler-Smith C (2007) Variation of 52 new Y-STR loci in the Y chromosome consortium worldwide panel of 76 diverse individuals. Int J Leg Med 121:124-127

Martinez-Cadenas C, Blanco-Verea A, Hernando B, Busby GB, Brion M, Carracedo A, Salas A, Capelli C (2016) The relationship between surname frequency and $\mathrm{Y}$ chromosome variation in Spain. Eur J Hum Genet 24:120-128

McEvoy B, Bradley DG (2006) Y-chromosomes and the extent of patrilineal ancestry in Irish surnames. Hum Genet 119:212-219

Mulero JJ, Chang CW, Calandro LM, Green RL, Li Y, Johnson CL, Hennessy LK (2006) Development and validation of the AmpFlSTR Yfiler PCR amplification kit: a male specific, single amplification 17 Y-STR multiplex system. J Forensic Sci 51:64-75

Phillips C (2015) Forensic genetic analysis of bio-geographical ancestry. Forensic Sci Int Genet 18:49-65

Purps J, Siegert S, Willuweit S, Nagy M, Alves C, Salazar R, Angustia SM, Santos LH, Anslinger K, Bayer B, Ayub Q, Wei W, Xue Y, Tyler-Smith C, Bafalluy MB, Martinez-Jarreta B, Egyed B, Balitzki B, Tschumi S, Ballard D, Court DS, Barrantes X, Bassler G, Wiest T, Berger B, Niederstatter H, Parson W, 
Davis C, Budowle B, Burri H, Borer U, Koller C, Carvalho EF, Domingues PM, Chamoun WT, Coble MD, Hill CR, Corach D, Caputo M, D'Amato ME, Davison S, Decorte R, Larmuseau MH, Ottoni C, Rickards O, Lu D, Jiang C, Dobosz T, Jonkisz A, Frank WE, Furac I, Gehrig C, Castella V, Grskovic B, Haas C, Wobst J, Hadzic G, Drobnic K, Honda K, Hou Y, Zhou D, Li Y, Hu S, Chen S, Immel UD, Lessig R, Jakovski Z, Ilievska T, Klann AE, Garcia CC, de Knijff P, Kraaijenbrink T, Kondili A, Miniati P, Vouropoulou M, Kovacevic L, Marjanovic D, Lindner I, Mansour I, Al-Azem M, Andari AE, Marino M, Furfuro S, Locarno L, Martin P, Luque GM, Alonso A, Miranda LS, Moreira H, Mizuno N, Iwashima Y, Neto RS, Nogueira TL, Silva R, Nastainczyk-Wulf M, Edelmann J, Kohl M, Nie S, Wang X, Cheng B et al (2014) A global analysis of Y-chromosomal haplotype diversity for 23 STR loci. Forensic Sci Int Genet 12:12-23

Purps J, Geppert M, Nagy M, Roewer L (2015) Validation of a combined autosomal/Y-chromosomal STR approach for analyzing typical biological stains in sexual-assault cases. Forensic Sci Int Genet 19:238-242

Ralf A, van Oven M, Zhong K, Kayser M (2015) Simultaneous analysis of hundreds of Y-chromosomal SNPs for high-resolution paternal lineage classification using targeted semiconductor sequencing. Hum Mutat 36:151-159

Rodig H, Roewer L, Gross A, Richter T, de Knijff P, Kayser M, Brabetz W (2008) Evaluation of haplotype discrimination capacity of 35 Y-chromosomal short tandem repeat loci. Forensic Sci Int 174:182-188

Roewer L (2009) Y chromosome STR typing in crime casework. Forensic Sci Med Pathol 5:77-84

Roewer L, Epplen JT (1992) Rapid and sensitive typing of forensic stains by PCR amplification of polymorphic simple repeat sequences in case work. Forensic Sci Int 53:163-171

Roewer L, Arnemann J, Spurr NK, Grzeschik KH, Epplen JT (1992) Simple repeat sequences on the human $\mathrm{Y}$ chromosome are equally polymorphic as their autosomal counterparts. Hum Genet 89:389-394

Roewer L, Kayser M, Dieltjes P, Nagy M, Bakker E, Krawczak M, de Knijff P (1996) Analysis of molecular variance (AMOVA) of Y-chromosome-specific microsatellites in two closely related human populations. Hum Mol Genet 5:1029-1033

Santos FR, Pandya A, Tyler-Smith C (1998) Reliability of DNAbased sex tests. Nat Genet 18:103

Scozzari R, Massaia A, Trombetta B, Bellusci G, Myres NM, Novelletto A, Cruciani F (2014) An unbiased resource of novel SNP markers provides a new chronology for the human $\mathrm{Y}$ chromosome and reveals a deep phylogenetic structure in Africa. Genome Res 24:535-544
Steinlechner M, Berger B, Niederstatter H, Parson W (2002) Rare failures in the amelogenin sex test. Int J Leg Med 116:117-120

Thangaraj K, Reddy AG, Singh L (2002) Is the amelogenin gene reliable for gender identification in forensic casework and prenatal diagnosis? Int J Leg Med 116:121-123

Thompson JM, Ewing MM, Frank WE, Pogemiller JJ, Nolde CA, Koehler DJ, Shaffer AM, Rabbach DR, Fulmer PM, Sprecher CJ, Storts DR (2013) Developmental validation of the PowerPlex(R) Y23 System: a single multiplex Y-STR analysis system for casework and database samples. Forensic Sci Int Genet 7:240-250

Trombetta B, D’Atanasio E, Massaia A, Ippoliti M, Coppa A, Candilio F, Coia V, Russo G, Dugoujon JM, Moral P, Akar N, Sellitto D, Valesini G, Novelletto A, Scozzari R, Cruciani F (2015) Phylogeographic refinement and large scale genotyping of human $\mathrm{Y}$ Chromosome haplogroup E provide new insights into the dispersal of early pastoralists in the African Continent. Genome Biol Evol 7:1940-1950

Underhill PA, Kivisild T (2007) Use of Y chromosome and mitochondrial DNA population structure in tracing human migrations. Annu Rev Genet 41:539-564

van Oven M, Ralf A, Kayser M (2011) An efficient multiplex genotyping approach for detecting the major worldwide human Y-chromosome haplogroups. Int J Leg Med 125:879-885

van Oven M, van den Tempel N, Kayser M (2012) A multiplex SNP assay for the dissection of human Y-chromosome haplogroup $\mathrm{O}$ representing the major paternal lineage in East and Southeast Asia. J Hum Genet 57:65-69

van Oven M, Toscani K, van den Tempel N, Ralf A, Kayser M (2013) Multiplex genotyping assays for fine-resolution subtyping of the major human Y-chromosome haplogroups E, G, I, J, and R in anthropological, genealogical, and forensic investigations. Electrophoresis 34:3029-3038

van Oven M, Van Geystelen A, Kayser M, Decorte R, Larmuseau MH (2014) Seeing the wood for the trees: a minimal reference phylogeny for the human Y chromosome. Hum Mutat 35:187-191

Vermeulen M, Wollstein A, van der Gaag K, Lao O, Xue Y, Wang Q, Roewer L, Knoblauch H, Tyler-Smith C, de Knijff P, Kayser M (2009) Improving global and regional resolution of male lineage differentiation by simple single-copy Y-chromosomal short tandem repeat polymorphisms. Forensic Sci Int Genet 3:205-213

Willuweit S, Roewer L (2015) The new Y Chromosome haplotype reference database. Forensic Sci Int Genet 15:43-48

Xue Y, Wang Q, Long Q, Ng BL, Swerdlow H, Burton J, Skuce C, Taylor R, Abdellah Z, Zhao Y, Asan MacArthur DG, Quail MA, Carter NP, Yang H, Tyler-Smith C (2009) Human Y chromosome base-substitution mutation rate measured by direct sequencing in a deep-rooting pedigree. Curr Biol 19:1453-1457 